\title{
Retorno ao esporte após osteotomia tibial alta com método de cunha de abertura*
}

\section{Return to Sports After High Tibial Osteotomy Using the Opening Wedge Technique}

\author{
Alexandre Pedro Nicolini ${ }^{1 \oplus}$ Eduardo Suñe Christiano ${ }^{1 \oplus}$ Rene Jorge Abdalla ${ }^{2}$ Moises Cohen ${ }^{2}$ \\ Rogério Teixeira de Carvalho ${ }^{30}$ \\ ${ }^{1}$ Centro de Traumatologia do Esporte, Escola Paulista de Medicina, \\ Universidade Federal de São Paulo, São Paulo, SP, Brasil \\ Endereço para correspondência Alexandre Pedro Nicolini, Centro de \\ Traumatologia do Esporte, Rua Estado de Israel, 636, Vila Clementino, \\ 2 Escola Paulista de Medicina, Universidade Federal de São Paulo, São \\ São Paulo, SP, 04022-001, Brasil (e-mail: apnicolini@uol.com.br).
}

Paulo, SP, Brasil

${ }^{3}$ Departamento de Ortopedia e Traumatologia, Instituto de Assistência

Médica ao Servidor Público Estadual de São Paulo, São Paulo, SP, Brasil

Rev Bras Ortop 2021;56(3):313-319.

\section{Resumo \\ Palavras-chave \\ - osteoartrose \\ - osteotomia \\ - esportes \\ - tíbia}

Abstract
Objetivo Avaliar o retorno ao esporte em pacientes jovens e ativos praticantes de alguma modalidade esportiva submetidos a osteotomia tibial alta (OTA) com o método de cunha de abertura.

Métodos Foram analisados prospectivamente 12 pacientes submetidos ao procedimento de OTA utilizando-se método de cunha de abertura. Todos os pacientes estavam afastados do esporte. Foram utilizados os escores Lysholm, questionário International Knee Documentation Committee (IKDC, na sigla em inglês), escala analógica de dor e nível de retorno em comparação ao período pré-operatório. O tempo médio de seguimento foi de 12 meses.

Resultados Um paciente retornou ao esporte em nível muito abaixo do pré-operatório, oito pacientes retornaram em nível pouco abaixo, dois pacientes retornaram no mesmo nível e um paciente retornou em nível acima.

Conclusão A OTA com uso do método de cunha de adição como forma de tratamento para osteoartrose medial isolada demonstra resultados clínicos e funcionais favoráveis e permite o retorno ao esporte.

Objective The present paper evaluates the resuming of physical activities by young, active patients who practiced some sport modality and underwent a high tibial osteotomy (HTO) using the opening wedge technique.
Trabalho realizado no Centro de Traumatologia do Esporte, Escola Paulista de Medicina, Universidade Federal de São Paulo, São Paulo, SP, Brasil. recebido

07 de Março de 2020

aceito

01 de Junho de 2020

Publicado on-line

Setembro 25, 2020
DOI https://doi.org/

10.1055/s-0040-1715514.

ISSN 0102-3616. (c) 2020. Sociedade Brasileira de Ortopedia e Traumatologia. All rights reserved.

This is an open access article published by Thieme under the terms of the Creative Commons Attribution-NonDerivative-NonCommercial-License, permitting copying and reproduction so long as the original work is given appropriate credit. Contents may not be used for commercial purposes, or adapted, remixed, transformed or built upon. (https://creativecommons.org/ licenses/by-nc-nd/4.0/)

Thieme Revinter Publicações Ltda., Rua do Matoso 170, Rio de Janeiro, RJ, CEP 20270-135, Brazil 


\author{
Keywords \\ - osteoarthrosis \\ - osteotomy \\ - sports \\ - tibia
}

\begin{abstract}
Methods A total of 12 patients submitted to HTO using the opening wedge technique were prospectively analyzed. All patients were not playing sports at that time. Pre- and postoperative Lysholm and International Knee Documentation Committee (IKDC) scores, visual analog scale for pain and performance level were compared. The average follow-up time was of 12 months.

Results One patient resumed sporting activities at a performance level significantly lower compared to the preoperative level, while eight patients returned at a slightly below level, two returned at the same level and one patient returned at a higher level in comparison with the preoperative period.

Conclusion For isolated medial osteoarthrosis treatment, HTO using the opening wedge technique has favorable clinical and functional results, allowing patients to resume their sporting activities.
\end{abstract}

\section{Introdução}

Ultimamente, tem ocorrido um aumento do número de adultos jovens que apresentam osteoartrose (OA) do joelho e que, pela idade e atividade funcional, são incompatíveis para a realização de artroplastia total de joelho (ATJ). ${ }^{1-4}$ Mais de $25 \%$ das pessoas $<70$ anos apresentam OA dos joelhos com expectativa de aumento exponencial futuramente. Atividades esportivas e recreacionais são muito importantes para esse público e a $\mathrm{OA}$ acaba limitando sua prática. ${ }^{5}$

A OA unicompartimental (OAUJ) acomete, preferencialmente, o compartimento femorotibial medial e possui graus variados de acometimento, segundo critérios radiográficos definidos por Ahlback. Os sintomas predominantes são a dor que piora com a carga e a deformidade angular progressiva.

Dentre as diversas formas de tratamento da OAUJ destacase a osteotomia tibial alta (OTA) indicada para paciente fisiologicamente jovens ( $<60$ anos), com OA medial isolada, amplitude de movimento (ADM) preservada, ausência de instabilidade ligamentar associada e sintomas mínimos femoropatelares e que apresentaram falha do tratamento conservador. Como contraindicações relativas para a realização da OTA: pacientes $>65$ anos, OA avançada, OA tricompartimental, artrite inflamatória, ADM diminuída $\left(<120^{\circ}\right)$, fumante, obesidade (índice de massa corpórea (IMC) $>30$ ), contratura em flexão $>5^{\circ} .{ }^{5}$

Existem três tipos principais de OTA: com cunha de abertura, com cunha de subtração e a cupuliforme. Existem várias vantagens da cunha de abertura em comparação com a cunha de fechamento: maior precisão e facilidade para correção nos planos coronal e sagital; não há necessidade de osteotomia da fíbula ou abordagem da articulação tibiofibular proximal (protegendo o nervo fibular) e preservação do estoque ósseo, facilitando a conversão para ATJ. As desvantagens da cunha de abertura: criação de um defeito ósseo, que, dependendo do tamanho, necessita o uso de enxerto ósseo, risco de pseudoartrose, possibilidade de perda da correção devido ao colapso da síntese, maior tempo sem carga e maior atenção ao slope tibial. ${ }^{3,4,6-8}$

A técnica foi descrita pela primeira vez em 1961 por Jackson et al. ${ }^{9}$ entretanto só se tornou popular a partir de
1965 quando Coventry ${ }^{10}$ promoveu a técnica para tratamento da OA medial associada a deformidade em varo. Desde então, ocorreram inúmeros avanços quanto à técnica, fixação e seleção dos pacientes, com diminuição das complicações e melhores resultados. ${ }^{3,4,6-8}$

A OTA visa diminuir a carga no compartimento afetado e transferi-la para o compartimento sadio, corrigindo a deformidade angular e promovendo a melhora sintomática do joelho afetado. ${ }^{1-3,7,8}$

Diante das excelentes taxas de sobrevida da OTA ${ }^{11-19}$ e dos riscos potenciais da ATJ, como afrouxamento, desgaste do material ou fraturas periprotéticas em pacientes praticantes de atividade de alto impacto, ${ }^{20}$ é recomendável optarse pela primeira em adultos jovens. Johnstone sugeriu que se a osteotomia for indicada para pacientes jovens e o mesmo possa estar apto para retorno ao trabalho e às atividades esportivas. A taxa de retorno ao esporte relatada em pacientes submetidos a ATJ é de apenas $20 \%{ }^{5}$

Embora a técnica da OTA esteja bem descrita, existem poucos estudos que analisam o nível de retorno ao esporte e o resultado clínico após a realização da OTA com cunha de abertura no adulto jovem com OAUJ. ${ }^{2,3,22}$

O objetivo do presente estudo consiste em avaliar o retorno ao esporte em pacientes jovens e ativos praticantes de alguma modalidade esportiva submetidos a OTA com cunha de abertura através dos escores Lysholm, International Knee Documentation Committee (IKDC, na sigla em inglês), escala analógica da dor e nível de retorno em comparação ao pré-operatório.

\section{Material e métodos}

O estudo foi realizado no Centro de Traumatologia do Esporte, no período de julho de 2017 até janeiro de 2018, com a aprovação do Comitê de Ética em Pesquisa da instituição.

\section{Descrição da Amostra}

Foram avaliados 12 pacientes com média de idade de 34,3 anos (30-44), 2 mulheres e 10 homens com IMC médio de $28,8 \mathrm{Kg} / \mathrm{m} 2(24,4-33,7 \mathrm{Kg} / \mathrm{m} 2)$. O grau de ADM no pré- 
operatório variou de $90^{\circ}$ até $132^{\circ}$ (média $108,3^{\circ}$ ) e o valor do eixo mecânico do membro inferior afetado variou de $6,4^{\circ}$ de varo até $2^{\circ}$ de valgo, com média de $3,4 \pm 2,2^{\circ}$ de varo.

Seis eram praticantes de corrida de rua (metragens variando de 5 a 21 quilômetros), quatro praticantes de futebol de campo, um de handebol e um praticante de artes marciais (artes marciais mistas [MMA, na sigla em inglês]).

Foram incluídos pacientes adultos com idades entre $20 \mathrm{e}$ 55 anos, com OA grau II e III segundo critérios de Ahlback, IMC $<35$, com $\mathrm{ADM}>90^{\circ}$ e afastamento do esporte há pelo menos 3 meses.

Foram excluídos pacientes submetidos a cirurgia prévia no joelho afetado; portadores de artrose bicompartimental ou tricompartimental; deformidade em varo $>15^{\circ}$; presença de instabilidade ligamentar sintomática; pacientes diabéticos, fumantes e pacientes com artrites inflamatórias.

\section{Descrição dos Procedimentos}

Todos os pacientes foram submetidos a tratamento clínico prévio por, no mínimo, 6 meses antes da realização da cirurgia. Todos os pacientes foram submetidos ao procedimento de OTA utilizando-se método de cunha de abertura com utilização de placa tipo Puddu $^{23}$ e submetidos ao mesmo protocolo de reabilitação.

Foi realizado o planejamento pré-operatório com a utilização de radiografias panorâmicas longas com análise dos eixos mecânico e anatômico, permitindo o cálculo do tamanho da cunha a ser executada, conforme o método descrito por Noyes e Dugdale. 24,25

A OTA foi realizada com o objetivo de se manter neutro o eixo de carga do membro inferior, associada à hipercorreção em torno de $2^{\circ}$ a $5^{\circ}$ de valgo, de modo que o eixo mecânico passe lateralmente ao centro da articulação do joelho, idealmente entre a porção média e lateral do côndilo lateral (62\%), discretamente lateral à eminência intercondilar lateral. ${ }^{3,24}$

Foram realizadas cunhas de abertura de até $15 \mathrm{~mm}$, sendo que até $10 \mathrm{~mm}$ utilizou-se somente o espaçador da placa e de 10 a $15 \mathrm{~mm}$ foi acrescentado enxerto autólogo tricortical da crista ilíaca ipsilateral.

A carga só foi liberada após consolidação radiográfica da fratura. Os questionários também foram aplicados em todos os casos antes da cirurgia e após 6 semanas, 3 meses, 6 meses e 12 meses de pós-operatório. Foram aplicados os questionários IKDC e Lysholm para avaliação do retorno ao esporte, atividades diárias, parâmetros clínicos e satisfação pessoal para cada paciente. Ainda foi aplicada a escala analógica de dor no período pré- e pós-operatório com 6 meses.

Através da avaliação de radiografias panorâmicas longas anteroposterior, foram registrados os valores do eixo anatômico (eixo femorotibial) e mecânico do membro inferior no pré-operatório e pós-operatório e o grau de artrose seguindo os critérios de Ahlback.

Foi questionado aos pacientes o nível de retorno ao esporte: não retornou ao esporte; retornou em nível muito abaixo do pré-operatório; retornou em nível pouco abaixo do pré-operatório; retornou em nível igual ao pré-operatório e retornou em nível superior ao pré-operatório.
Na análise estatística, utilizou-se a correlação de Spearman para medir o grau de relação entre todas as variáveis quantitativas em todos os momentos: arco de movimento, eixo anatômico, escala analógica de dor, tempo de consolidação, índice de Lysholm, pontuação no IKDC e IMC.

O teste de Mann-Whitney foi utilizado para comparar o grau de artrose para algumas variáveis quantitativas: eixo anatômico pré-operatório, ganho de pontos na escala analógica de dor, índice Lysholm, resultado do questionário IKDC e tempo de consolidação. Os resultados foram expressos como média e desvio padrão (DP).

\section{Resultados}

Observou-se correção do eixo anatômico: variação de $3,6^{\circ}$ a $9^{\circ}$ de valgo (média $6,38 \pm 1,4^{\circ}$ ). 0 tempo de consolidação radiográfica variou de 7 a 11 semanas (média 9,16 semanas). Este valor foi correlacionado com os sintomas do paciente e 0 tempo no qual foi iniciada a carga parcial progressiva.

Em relação ao escore de Lysholm, a pontuação média dos 12 pacientes estudados foi de $83,8 \pm 8,2$ pontos, do qual houve uma variação entre 70 e 94 pontos $(p<005)$. Nesta avaliação, 7 pacientes (58,33\%) obtiveram resultados bons e 5 pacientes $(41,66 \%)$ apresentaram resultados regulares.

Avaliando-se o questionário IKDC após a padronização para o sistema percentil, obteve-se uma média de 75,8 $\pm 9,8$ com variação entre $62 \%$ e $84 \%(p<0,05)$.

Um dos pacientes evoluiu com retardo de consolidação, sendo necessário novo procedimento para colocação de enxerto autólogo após 6 meses do procedimento inicial, evoluindo satisfatoriamente após o período de 1 ano do procedimento inicial.

Dos pacientes estudados, um paciente retornou ao esporte em nível muito abaixo do pré-operatório, oito pacientes retornaram em nível pouco abaixo do pré-operatório, dois pacientes retornaram no mesmo nível do pré-operatório e um paciente retornou em nível acima do pré-operatório.

Comparando-se o ganho de ADM e escala analógica de dor no pré- e pós-operatório, verificamos que existe diferença estatisticamente significante entre os momentos para as duas variáveis. A média da escala analógica de dor caiu de 6,83 para 3,53 e a média de ADM aumentou de $108,33^{\circ}$ para $123,92^{\circ}(\boldsymbol{p}<0,001)$ (-Tabela $\mathbf{1}$ ).

Utilizou-se a correlação de Spearman para medir o grau de relação entre todas as variáveis quantitativas em todos os momentos. $O$ teste de correlação foi utilizado para validar as correlações e os valores foram expressos em porcentagem. Nesta escala, considera-se o valor de 0 a $20 \%$ como uma correlação péssima; 20 a $40 \%$ ruim; 40 a $60 \%$ regular; 60 a $80 \%$ boa e 80 a $100 \%$ ótima.

Através dessa análise ( - Tabela 2), verificou-se que existem algumas correlações estatisticamente significantes: entre eixo anatômico pré e o escore Lysholm no valor de $+65,4 \%$, ou seja, quanto maior a deformidade no pré-operatório, melhor foi o resultado obtido no escore subjetivo de Lysholm; entre eixo anatômico pré e o ganho na escala analógica de dor $+48,5 \%$; entre o eixo anatômico pré e o resultado do questionário IKDC $+48,5 \%$. Uma correlação inversamente proporcional 
Tabela 1 Comparação entre momentos pré-operatório e pós-operatório

\begin{tabular}{|l|l|l|l|l|l|l|l|l|l|}
\hline \multicolumn{2}{|l|}{ Momento } & Média & Mediana & Desvio Padrão & Q1 & Q3 & $\boldsymbol{n}$ & IC & Valor-p \\
\hline \multirow{2}{*}{ Arco de movimento } & Pré & 108,33 & 105,0 & 12,64 & 101,5 & 115,0 & 12 & 7,15 & $<0,001$ \\
\cline { 2 - 9 } & Pós & 123,92 & 124,0 & 7,49 & 119,5 & 130,0 & 12 & 4,24 & \\
\hline \multirow{2}{*}{ Eixo anatomico } & Pré & $-3,32$ & $-3,9$ & 2,75 & $-5,4$ & $-2,0$ & 12 & 1,56 & $<0,001$ \\
\cline { 2 - 9 } & Pós & 6,38 & 6,5 & 1,89 & 4,8 & 8,0 & 12 & 1,07 & \\
\hline \multirow{2}{*}{ Escala analógica dor } & Pré & 6,83 & 6,4 & 1,12 & 6,0 & 7,9 & 12 & 0,64 & $<0,001$ \\
\cline { 2 - 8 } & Pós & 3,53 & 3,4 & 0,77 & 3,1 & 4,1 & 12 & 0,44 & \\
\hline
\end{tabular}

Abreviações: IC, intervalo de confiança; Pós, pós-operativo; Pré, pré-operativo.

entre o eixo anatômico pré e o ganho de ADM - 42,4\%, ou seja, quanto maior era a deformidade no pré-operatório, menor foi o ganho de ADM $(p<0,001)$.

Outras correlações observadas que também merecem destaque foram entre o valor do IMC e o resultado do IKDC - $58,8 \%$ e entre a ADM no pré-operatório e o resultado do IKDC $+60,8$.

Através do teste de Mann-Whitney, concluiu-se que não existe diferença estatisticamente significante entre os graus de artrose para as variáveis analisadas: eixo anatômico préoperatório, ganho de pontos na escala analógica de dor, Lysholm, IKDC e tempo de consolidação ( - Tabela 3 ).

\section{Discussão}

Há diversos questionamentos quanto ao resultado e a capacidade dos pacientes submetidos ao procedimento de OTA retornarem efetivamente à prática de esportes, além da possibilidade de praticar as atividades de vida diária e recreacionais sem limitações e sem dor.

A OTA com cunha de abertura para tratamento de OAUJ e mau alinhamento apresenta excelentes ou bons resultados na literatura: Hernigou et al. ${ }^{13}$ relataram $81 \%$ de resultados excelentes ou bons após 10 anos de seguimento com 53 pacientes. Aglietti et al. ${ }^{26}$ acompanharam 61 pacientes clinicamente até 21 anos após realização de OTA e observaram que $79 \%$ deles não tiveram nenhuma dor ou apresentavam dor leve no joelho operado.

Em relação à prática desportiva após OTA, alguns estudos prévios da literatura mostraram bons resultados com altas taxas de retorno. Salzmann et al. ${ }^{22}$ encontraram em sua amostra que $90,9 \%$ dos pacientes estavam participando de esportes e atividades recreativas, comparado com $87,9 \%$ antes da cirurgia. 0 índice de Lysholm e a escala visual analógica de dor aumentaram significativamente, de 42,4 para 69,6 e de 6,9 para 2,9 , respectivamente $(p<0,01)$.

Faschingbauer et al. ${ }^{21}$ analisaram a taxa de retorno ao trabalho e ao esporte em 51 pacientes submetidos a OTA. Segundo os autores, 92.3\% retornaram aos níveis de atividade física semelhantes ao pré-operatório e observaram uma troca de atividades de alto impacto para atividades de baixo impacto. Além disso, notaram uma diminuição na duração da atividade esportiva e na quantidade das modalidades esportivas.

Hoorntje et al. ${ }^{27}$ realizaram uma revisão sistemática sobre o tema e encontraram uma taxa de retorno ao esporte em estudos com boa metodologia e baixo risco de viés (11 estudos) de $82 \%$. Também reportaram uma taxa de sobrevivência de 87 a $99 \%$ com 5 anos e de 66 a $84 \%$ com 10 anos após OTA. Os estudos diferiram bastante quanto à avaliação da atividade esportiva (nível de prática).

Ekhtiari et al. $^{28}$ realizaram outra revisão sistemática visando analisar o retorno ao trabalho e ao esporte após OTA. Incluíram 11 estudos com 250 pacientes com idade média de 46,2 anos. 0 método de cunha de abertura foi o mais utilizado. Um total de $87,2 \%$ dos pacientes retornou à atividade esportiva e 78,6\% retornaram no mesmo nível ou nível acima. Entre os atletas competitivos, 54\% retornaram às competições. Aproximadamente $90 \%$ dos pacientes que retornaram ao trabalho e atividades esportivas o fizeram dentro de 1 ano. Um total de $7 \%$ foram submetidos a ATJ com uma média de tempo de 6,7 anos. Vários métodos foram utilizados para mensurar o nível de atividade física.

Em outro estudo com essa mesma temática, Bastard et al. ${ }^{29}$ analisaram retrospectivamente 30 pacientes com seguimento médio de 1,3 anos e observaram que todos os pacientes retornaram ao esporte dentro de um ano, 73,3\% no nível pré-cirurgia e $23,3 \%$ em nível acima.

W-Dahl et al. ${ }^{30}$ acompanharam por 10 anos 79 pacientes submetidos a OTA. Após 10 anos, 25 pacientes foram submetidos a ATJ. Os autores concluíram que é uma excelente solução para pacientes jovens com $\mathrm{OA}$, apresentando moderada degeneração ao longo do tempo e propiciando a possibilidade de atividade física e qualidade de vida. Após 2 anos da OTA, os pacientes aumentaram sua atividade física, com mais da metade ativo em esportes como golfe, dança, caminhada e hidroginástica. Após 10 anos, quase metade ainda se encontrava ativa nas mesmas atividades.

Nosso estudo corrobora esses achados e demonstra que há bons resultados funcionais após a OTA com cunha de abertura. Comparando-se a correção do eixo anatômico, observamos que houve correção satisfatória de $3,31 \pm 1,2^{\circ}$ de varo para média de $6,38 \pm 1,8^{\circ}$ de valgo. Um dos pacientes não obteve a hipercorreção, e nesse caso houve um alinhamento para $3,6^{\circ}$ de valgo. Os piores resultados funcionais são referentes a este paciente com pontuação de Lysholm 70 e IKDC 62\%. Deve-se enfatizar a importância do planejamento pré-operatório adequado, cálculo do eixo anatômico e mecânico e programação do tamanho da cunha a ser executada, que repercutirá diretamente no resultado esperado. 
Retorno ao esporte após osteotomia tibial alta Nicolini et al. 317

Tabela 2 Correlação das variáveis

\begin{tabular}{|c|c|c|c|c|c|c|c|c|c|c|c|c|c|}
\hline & & \multirow[b]{2}{*}{ Pré } & \multirow{2}{*}{$\begin{array}{l}\text { ADM } \\
\text { Pós }\end{array}$} & \multirow[b]{2}{*}{ Ganho } & \multicolumn{2}{|c|}{ Eixo anatômico } & \multirow[b]{2}{*}{ Ganho } & \multicolumn{3}{|c|}{ Escala Analógica Dor } & \multirow{2}{*}{$\begin{array}{l}\text { Tempo } \\
\text { de } \\
\text { consoli- } \\
\text { dação }\end{array}$} & \multirow{2}{*}{$\begin{array}{l}\text { Índice } \\
\text { Lysholm }\end{array}$} & \multirow{2}{*}{$\begin{array}{l}\text { Índice } \\
\text { IKDC }\end{array}$} \\
\hline & & & & & Pré & Pós & & Pré & Pós & Ganho & & & \\
\hline Pós & Corr & $76.50 \%$ & & & & & & & & & & & \\
\hline ADM & valor-p & 0.004 & & & & & & & & & & & \\
\hline \multirow[t]{2}{*}{ Ganho } & Corr & $-67.00 \%$ & $-19.70 \%$ & & & & & & & & & & \\
\hline & valor-p & 0.017 & 0.539 & & & & & & & & & & \\
\hline \multirow[t]{2}{*}{ Pré } & Corr & $57.90 \%$ & $58.00 \%$ & $-42.40 \%$ & & & & & & & & & \\
\hline & valor-p & 0.049 & 0.048 & 0.169 & & & & & & & & & \\
\hline \multicolumn{14}{|l|}{ Eixo } \\
\hline Pós & Corr & $21.40 \%$ & $11.30 \%$ & $-8.70 \%$ & $35 \%$ & & & & & & & & \\
\hline Anatômico & valor-p & 0.503 & 0.727 & 0.789 & 0.264 & & & & & & & & \\
\hline \multirow[t]{2}{*}{ Ganho } & Corr & $-56.10 \%$ & $-74.50 \%$ & $32.70 \%$ & $-87.90 \%$ & $-4.60 \%$ & & & & & & & \\
\hline & valor-p & 0.058 & 0.005 & 0.299 & $<0,001$ & 0.888 & & & & & & & \\
\hline \multirow[t]{2}{*}{ Pré } & Corr & $7.80 \%$ & $-3.40 \%$ & $-19.80 \%$ & $-32.50 \%$ & $4.40 \%$ & $21.20 \%$ & & & & & & \\
\hline & valor-p & 0.810 & 0.917 & 0.538 & 0.303 & 0.892 & 0.506 & & & & & & \\
\hline \multicolumn{14}{|l|}{$\begin{array}{l}\text { Escala } \\
\text { analógica }\end{array}$} \\
\hline Pós & Corr & $7.20 \%$ & $-7.80 \%$ & $-20.90 \%$ & $3.20 \%$ & $-43.80 \%$ & $-1.20 \%$ & $17.20 \%$ & & & & & \\
\hline dor & valor-p & 0.824 & 0.811 & 0.514 & 0.922 & 0.155 & 0.97 & 0.594 & & & & & \\
\hline \multirow[t]{2}{*}{ Ganho } & Corr & $0.90 \%$ & $-6.10 \%$ & $-5.30 \%$ & $48.50 \%$ & $-3.90 \%$ & $-27.50 \%$ & $-87.90 \%$ & $15.70 \%$ & & & & \\
\hline & valor-p & 0.978 & 0.874 & 0.869 & 0.110 & 0.905 & 0.388 & $<0,001$ & 0.626 & & & & \\
\hline \multirow{2}{*}{$\begin{array}{l}\text { Tempo de } \\
\text { consolidação }\end{array}$} & Corr & $50.80 \%$ & $61.20 \%$ & $-37.60 \%$ & $34.40 \%$ & $-47.50 \%$ & $-58.30 \%$ & $-19.90 \%$ & $24.60 \%$ & $19.60 \%$ & & & \\
\hline & valor-p & 0.092 & 0.035 & 0.228 & 0.274 & 0.119 & 0.047 & 0.536 & 0.441 & 0.541 & & & \\
\hline \multirow{2}{*}{$\begin{array}{l}\text { Índice } \\
\text { Lysholm }\end{array}$} & Corr & $64.60 \%$ & $52.80 \%$ & $-52.90 \%$ & $65.40 \%$ & $11.10 \%$ & $-67.40 \%$ & $9.80 \%$ & $33.70 \%$ & $9.00 \%$ & $30.80 \%$ & & \\
\hline & valor-p & 0.023 & 0.077 & 0.077 & 0.021 & 0.731 & 0.016 & 0.763 & 0.284 & 0.781 & 0.331 & & \\
\hline \multirow{2}{*}{$\begin{array}{l}\text { Índice } \\
\text { IKDC }\end{array}$} & Corr & $60.80 \%$ & $53.40 \%$ & $-23.80 \%$ & $43.90 \%$ & $45.60 \%$ & $-36.40 \%$ & $44.90 \%$ & $-3.70 \%$ & $-32.00 \%$ & $-6.50 \%$ & $54.90 \%$ & \\
\hline & valor-p & 0.036 & 0.074 & 0.456 & 0.153 & 0.136 & 0.244 & 0.144 & 0.909 & 0.311 & 0.842 & 0.064 & \\
\hline \multirow[t]{2}{*}{ IMC } & Corr & $-39.20 \%$ & $-45.20 \%$ & $-12.00 \%$ & $-52.20 \%$ & $-51.90 \%$ & $34.60 \%$ & $7.10 \%$ & $14.20 \%$ & $-5.10 \%$ & $12.70 \%$ & $-12.00 \%$ & $-68.80 \%$ \\
\hline & valor-p & 0.208 & 0.140 & 0.709 & 0.082 & 0.084 & 0.271 & 0.827 & 0.659 & 0.875 & 0.693 & 0.711 & 0.044 \\
\hline
\end{tabular}

Abreviações: ADM, arco de movimento; Corr, correlação; IKDC, International Knee Documentation Committee; Pós, pós-operatório; Pré, préoperatório.

Tabela 3 Comparação de grau de artrose

\begin{tabular}{|l|l|l|l|l|l|l|l|l|l|}
\hline \multicolumn{2}{|l|}{ Grau de Artrose (Ahlback) } & Média & Mediana & Desvio Padrão & Q1 & Q3 & $\boldsymbol{n}$ & IC & valor-p \\
\hline \multirow{2}{*}{ Eixo anatômico pré-operatório } & Grau II & $-2,80$ & $-2,2$ & 3,55 & $-6,2$ & $-1,2$ & 5 & 3,11 & 0,808 \\
\cline { 2 - 9 } & Grau III & $-3,69$ & $-4,2$ & 2,25 & $-4,8$ & $-3,5$ & 7 & 1,67 & \\
\hline \multirow{2}{*}{ Dor Ganho } & Grau II & $-3,48$ & $-4,0$ & 0,98 & $-4,0$ & $-3,0$ & 5 & 0,86 & 0,935 \\
\cline { 2 - 9 } & Grau III & $-3,17$ & $-3,2$ & 1,34 & $-4,2$ & $-2,5$ & 7 & 0,99 & \\
\hline \multirow{2}{*}{ Indice Lysholm } & Grau II & 82,00 & 82,0 & 9,27 & 76,0 & 90,0 & 5 & 8,13 & 0,624 \\
\cline { 2 - 9 } & Grau III & 85,14 & 88,0 & 8,07 & 80,0 & 90,0 & 7 & 5,98 & \\
\hline \multirow{2}{*}{ Indice IKDC } & Grau II & 78,40 & 80,0 & 5,18 & 74,0 & 82,0 & 5 & 4,54 & 0,414 \\
\cline { 2 - 9 } & Grau III & 74,00 & 76,0 & 8,16 & 69,0 & 79,0 & 7 & 6,05 & \\
\hline \multirow{2}{*}{ Tempo de consolidação } & Grau II & 9,40 & 10,0 & 1,34 & 8,0 & 10,0 & 5 & 1,18 & 0,618 \\
\cline { 2 - 8 } & Grau III & 9,00 & 9,0 & 1,29 & 8,5 & 9,5 & 7 & 0,96 & \\
\hline
\end{tabular}

Abreviações: IC, índice de confiança; IKDC, International Knee Documentation Committee. 
Analisando-se especificamente os resultados do índice de Lysholm e o questionário subjetivo IKDC, foi observado um resultado significante, sendo que 7 pacientes $(58,33 \%)$ apresentaram resultado bom de Lysholm e para IKDC média percentual de 75,8 $\pm 9,8(p<0,05)$ com índice que alcançou $84 \%$.

Houve uma melhora significante no ganho de ADM e na pontuação referente à escala analógica de dor. O presente estudo reforça o uso da OTA com cunha de abertura com obtenção de bons resultados e a possibilidade do retorno ao nível de atividade próximo ao nível pré-operatório.

O presente estudo apresenta algumas limitações: amostra pequena; falta de grupo controle; tempo de seguimento curto que pode interferir no nível de retorno ao esporte e falta de avaliação da periodicidade, intensidade e volume de treino. $O$ estudo apresenta os resultados preliminares e visa acrescentar mais pacientes para avaliação futura e resultado estatístico mais significante.

\section{Conclusão}

A OTA de cunha de adição como forma de tratamento para OA medial isolada demonstra resultados clínicos e funcionais favoráveis e permite o retorno ao esporte.

\section{Declarações}

- Aprovado pelo Comitê de Ética da UNIFESP.

- Todos os participantes assinaram termo de consentimento livre e esclarecido.

- Todo o material / dados analisados estão incluídos no artigo.

- Suporte financeiro.

Sem suporte financeiro. Todo o custo para a coleta, análise, interpretação dos resultados e escrita foram providenciados exclusivamente pelos autores.

\section{Contribuição dos autores}

Nicolini A. P. foi responsável pela concepção e desenho, aquisição dos pacientes do estudo, análise e interpretação dos dados, redação do artigo e aprovação final da versão submetida.

Christiano E. S. foi responsável pela concepção e design, suporte logístico, redação do artigo e aprovação final da versão submetida.

Cohen M. foi responsável pela concepção e design, redação do artigo, perícia estatística, suporte técnico e aprovação final da versão submetida.

Abdallah R. J. foi responsável pela concepção e desenho, redação do artigo e aprovação final da versão submetida. Carvalho R. T. foi responsável pela concepção e desenho, análise e interpretação dos dados, redação do artigo, revisão crítica, aquisição dos pacientes do estudo, redação do artigo e aprovação final da versão submetida.

\section{Conflitos de Interesse}

Os autores declaram não haver conflitos de interesse.

\section{Referências}

1 Shim JS, Lee SH, Jung HJ, Lee HI. High tibial open wedge osteotomy below the tibial tubercle: clinical and radiographic results. Knee Surg Sports Traumatol Arthrosc 2013;21(01):57-63

2 Wolcott M, Traub S, Efird C. High tibial osteotomies in the young active patient. Int Orthop 2010;34(02):161-166

3 Avakian R, Severino NR, Cury RPL, Oliveira VM, Aihara T, Camargo OPA. High Tibial Osteotomy In Patients With Knee Arthrosis. Acta Ortop Bras 2008;16(03):152-156

4 Gomoll AH. High tibial osteotomy for the treatment of unicompartmental knee osteoarthritis: a review of the literature, indications, and technique. Phys Sportsmed 2011;39(03):45-54

5 Johnstone SF, Tranovich MJ, Vyas D, Wright VJ. Unicompartmental arthritis in the aging athlete: osteotomy and beyond. Curr Rev Musculoskelet Med 2013;6(03):264-272

6 Day M, Wolf BR. Medial Opening-Wedge High Tibial Osteotomy for Medial Compartment Arthrosis/Overload. Clin Sports Med 2019;38(03):331-349

7 Mello Junior WA, Arruda LRP, Coluccini AM, et al. Complicações da osteotomia em cunha de abertura medial do joelho: estudo retrospectivo. Rev Bras Ortop 2011;46(01):64-68

8 Saggin JI, Severo A, Borges JLP. Osteotomia valgizante proximal da tíbia no tratamento da osteoartrose. Rev Bras Ortop 1996;31(05): 383-388

9 Jackson JP, Waugh W, Green JP. High tibial osteotomy for osteoarthritis of the knee. J Bone Joint Surg Br 1969;51(01): 88-94

10 Coventry MB. Osteotomy about the knee for degenerative and rheumatoid arthritis. J Bone Joint Surg Am 1973;55(01):23-48

11 Bouharras M, Hoet F, Watillon M, et al. [Results of tibial valgus osteotomy for internal femoro-tibial arthritis with an average 8year follow-up]. Acta Orthop Belg 1994;60(02):163-169

12 Flecher X, Parratte S, Aubaniac JM, Argenson JN. A 12-28-year followup study of closing wedge high tibial osteotomy. Clin Orthop Relat Res 2006;452(452):91-96

13 Hernigou P, Medevielle D, Debeyre J, Goutallier D. Proximal tibial osteotomy for osteoarthritis with varus deformity. A ten to thirteen-year follow-up study. J Bone Joint Surg Am 1987;69 (03):332-354

14 Ivarsson I, Myrnerts R, Gillquist J. High tibial osteotomy for medial osteoarthritis of the knee. A 5 to 7 and 11 year follow-up. J Bone Joint Surg Br 1990;72(02):238-244

15 Lootvoet L, Massinon A, Rossillon R, Himmer O, Lambert K, Ghosez JP. [Upper tibial osteotomy for gonarthrosis in genu varum. Apropos of a series of 193 cases reviewed 6 to 10 years later]. Rev Chir Orthop Repar Appar Mot 1993;79(05):375-384

16 Majima T, Yasuda K, Katsuragi R, Kaneda K. Progression of joint arthrosis 10 to 15 years after high tibial osteotomy. Clin Orthop Relat Res 2000;(381):177-184

17 Rinonapoli E, Mancini GB, Corvaglia A, Musiello S. Tibial osteotomy for varus gonarthrosis. A 10- to 21-year followup study. Clin Orthop Relat Res 1998;(353):185-193

18 Rudan JF, Simurda MA. Valgus high tibial osteotomy. A long-term follow-up study. Clin Orthop Relat Res 1991;(268):157-160

19 Yasuda K, Majima T, Tsuchida T, Kaneda K. A ten- to 15-year followup observation of high tibial osteotomy in medial compartment osteoarthrosis. Clin Orthop Relat Res 1992;(282):186-195

20 Healy WL, Sharma S, Schwartz B, Iorio R. Athletic activity after total joint arthroplasty. J Bone Joint Surg Am 2008;90(10):2245-2252

21 Faschingbauer M, Nelitz M, Urlaub S, Reichel H, Dornacher D. Return to work and sporting activities after high tibial osteotomy. Int Orthop 2015;39(08):1527-1534

22 Salzmann GM, Ahrens P, Naal FD, et al. Sporting activity after high tibial osteotomy for the treatment of medial compartment knee osteoarthritis. Am J Sports Med 2009;37(02):312-318

23 Gomes JLE, Ruthner RP, Marczyk LRS. Osteotomia valgizante de tíbia com placa "calço" de Puddu: apresentação de técnica. Acta Ortop Bras 2000;8(03):134-139 
24 Dugdale TW, Noyes FR, Styer D. Pre-operative planning for high tibial osteotomy The effect of lateral tibiofemoral separation and tibiofemoral length. Clin Orthop 1992;2(47): 248-264

25 Olin MD, Vail TP. High tibial osteotomy: will new techniques provide better results? Curr Opin Orthop 2001;12(01):8-12

26 Aglietti P, Rinonapoli E, Stringa G, Taviani A. Tibial osteotomy for the varus osteoarthritic knee. Clin Orthop Relat Res 1983; 176:239-251

27 Hoorntje A, Witjes S, Kuijer PPFM, et al. High Rates of Return to Sports Activities and Work After Osteotomies Around the Knee: A Systematic Review and Meta-Analysis. Sports Med 2017;47(11): 2219-2244
28 Ekhtiari S, Haldane CE, de Sa D, Simunovic N, Musahl V, Ayeni OR. Return to Work and Sport Following High Tibial Osteotomy: A Systematic Review. J Bone Joint Surg Am 2016;98(18): 1568-1577

29 Bastard C, Mirouse G, Potage D, et al. Return to sports and quality of life after high tibial osteotomy in patients under 60 years of age. Orthop Traumatol Surg Res 2017;103(08):1189-1191

30 W-Dahl A, Toksvig-Larsen S, Lindstrand A. Ten-year results of physical activity after high tibial osteotomy in patients with knee osteoarthritis. Knee Surg Sports Traumatol Arthrosc 2017;25(03): 902-909 